\title{
Prevalence of Migraine Headache in Taif City, Saudi Arabia
}

\author{
Ziyad Atiah Almalki ${ }^{\mathrm{a}, \mathrm{e}}$, Mohammed Abdullah Ghramah Alzhrani ${ }^{\mathrm{a}}$, Asmaa Turki Altowairqia \\ Yara Abdulaziz Aljawi ${ }^{\mathrm{a}}$, Sarah Abdulaziz Fallatah ${ }^{\mathrm{b}}$, Luai Mohammed Assaedic, \\ Mohammed Abdulaziz Aljawia ${ }^{\text {, Sadeem Adnan Alqusair }}{ }^{\mathrm{d}}$
}

\begin{abstract}
Background: Migraine is a public health problem and it is the third most common cause of disability among individuals below the age of 50. In spite of the multiplicity of the studies concerned with exploring the disease epidemiology and nature worldwide, data from Saudi Arabia are lacking. The aim of this study was to study the prevalence of migraine in Taif city, Saudi Arabia.
\end{abstract}

Methods: This was a cross-sectional study conducted on 354 individuals in Taif city. Data were collected via a self-administered questionnaire including demographic variables, clinical variables, and therapeutic variables. Data were fed into the computer and analyzed using SPSS software.

Results: Among the 354 recruited participants, 54\% were females, and $46 \%$ were males. Migraine occurred in $89.9 \%$ of urban inhabitants. About $24 \%$ of migraineurs were unemployed, and $16 \%$ were non-working students. Headache occurred in $86.9 \%$ of females and $71.6 \%$ of males $(\mathrm{P}=0.002)$. Gender was significantly correlated with migraine prevalence (odds ratio $(\mathrm{OR})=0.28$ ). About $47 \%$ reported pain score of $4-6$. Seeking medical advice was encountered in $32.4 \%$ of females and $26.6 \%$ of males. Nausea occurred in more than onethird of patients before and during headache. Only $31.4 \%$ of participants knew about migraine triggers. Family history was found in $64 \%$ of participants.

Conclusions: Migraine is prevalent in Taif city. It is more prevalent among females, and among urban areas. Migraine is associated with high rates of unemployment. Most Saudi migraineurs encounter throbbing, moderately severe headache, that does not awake them from sleep. Females report more severe headaches, whereas more men take medications. Nausea is the most common associated symptom with migraine. Only one-third of Saudi migraineurs know about migraine triggers. Family history of migraine is very common among Saudi migraineurs.

Manuscript submitted November 24, 2017, accepted December 9, 2017

${ }^{a}$ College of Medicine, Taif University, Taif, KSA

bepartment of Family Medicine, King Abdulaziz Hospital, Jeddah, KSA

${ }^{\mathrm{c} C o l l e g e ~ o f ~ M e d i c i n e, ~ U m m ~ A l-Q u r a ~ U n i v e r s i t y, ~ M e c c a, ~ K S A ~}$

${ }^{\mathrm{d} C o l l e g e ~ o f ~ M e d i c i n e, ~ K i n g ~ A b d u l a z i z ~ U n i v e r s i t y, ~ J e d d a h, ~ K S A ~}$

${ }^{e}$ Corresponding Author: Ziyad Atiah Almalki, College of Medicine, Taif University, Taif, KSA. Email: z.a.alm@hotmail.com

doi: https://doi.org/10.14740/jocmr3277w
Keywords: Migraine; Taif; Prevalence

\section{Introduction}

Migraine is estimated to be the third cause of disability, according to the Global Burden Disease (GBD) 2015, in individuals under the age of 50 [1]. It can be severe enough to impair quality of life and daily activities. The most accepted pathophysiological hypothesis for migraine is the trigeminovascular theory. Trigeminal nerve fibers surrounding basal meningeal and cerebral vessels are thought to be stimulated, and subsequently release various inflammatory mediators such as vasoinhibitory peptide (VIP), calcitonin gene-related peptide (CGRP), and substance P. These substance cause local neurogenic inflammation, provoke vasodilatation, and further stimulate other nerve fibers. Pain impulses are transmitted centrally to the thalamus and sensory cortex [2].

Common migraine triggers include long fasting, hypoglycemia, sleep disturbance, psychological stress, hormonal changes such as oral contraceptive pills, menstruation, and ovulation), and some medications (such as nitroglycerin, reserpine, and estrogen) [2]. Migraine can occur with aura (typical migraine) or without aura (classical migraine). Migraine can also evolve through many stages, namely prodrome, aura, headache attack, and post prodrome [3]. The prodrome usually precedes the headache by up to 1 day. During this stage, the patient may experience irritability, mood swings, appetite changes, anorexia or food craving, sleep disturbances, and salt and water retention. It is commonly encountered among patients with typical migraine (migraine with aura). Migraine aura represents a focal cerebral dysfunction that precedes the headache onset in $10-20 \%$ in migraineurs [4]. It usually lasts shortly for 5 - 20 min on average. Migraine aura may be visual (e.g. blurred vision, fortification spectra, scotomata, scintillations, micropsia, macropsia, or black dots), sensory, motor, autonomic, cognitive, behavioral, or dizziness. The headache stage usually last for hours and may continue to several days (status migrainosus) [5]. It is typically severe and interfere with daily activities and, sometimes, with sleep. During the post prodrome stage, the migraineur feel exhausted and lethargic, or less commonly energized and elated [3].

Migraine is a public health problem that has been extensively studied during the past decades. It is prevalent among adolescence and young adults. In spite of the multiplicity of 
Table 1. The IHS Criteria for Migraine [6]

Migraine without aura

A. At least five attacks fulfilling criteria B-D.

B. Headache attacks lasting $4-72 \mathrm{~h}$ (untreated or unsuccessfully treated)

C. Headache has at least two of the following four characteristics:

1. Unilateral location

2. Pulsating quality

3. Moderate or severe pain intensity

4. Aggravation by or causing avoidance of routine physical activity (e.g., walking or climbing stairs)

D. During headache at least one of the following:

1. Nausea and/or vomiting

2. Photophobia and phonophobia

E. Not better accounted for by another ICHD-3 diagnosis.

Migraine with aura

A. At least two attacks fulfilling criteria $\mathrm{B}$ and $\mathrm{C}$

B. One or more of the following fully reversible aura symptoms:

1. Visual

2. Sensory

3. Speech and/or language

4. Motor

5. Brainstem

6. Retinal

C. At least two of the following four characteristics:

1. At least one aura symptom spreads gradually over $\geq 5 \mathrm{~min}$, and/or two or more symptoms occur in succession

2. Each individual aura symptom lasts 5 - $60 \mathrm{~min}$

3. At least one aura symptom is unilateral

4. The aura is accompanied, or followed within $60 \mathrm{~min}$, by headache

D. Not better accounted for by another ICHD-3 diagnosis, and transient ischemic attack has been excluded

the studies addressing the prevalence of migraine in different countries, local studies in Saudi Arabia are limited, and studies from Taif city are sparse. Thus, this study was conducted.

\section{Patients and Methods}

\section{Subjects and study design}

This is a cross-sectional study using a pre-designed questionnaire conducted through an online survey during August 2017. The survey was approved by the ethical board of Taif University, and the survey was undertaken among the patients suffering from headache in Taif city, Saudi Arabia.

\section{International Headache Society (IHS) criteria}

It is not feasible to diagnose migraine on basis of clinical examination in a population-based survey with large number in- cluded. The advantage of IHS criteria in such cases is that they have a standardized and structured method for integrating a medical history as a base for diagnosis. The IHS categories for migraine are migraine without aura (IHS 1.1) and migraine with aura (IHS 1.2) [6]. The IHS criteria for migraine are presented in Table 1. For patients to be classified as a migraineur respondent, they must have experienced five or more headaches in their lifetime fulfilling the various criteria indicated in Table 1.

We conducted a cross-sectional survey study using a prespecified questionnaire, distributed as hard copies by research collaborators. Our questionnaire was structured by a series of questions about headaches and related symptoms to finally determine whether the respondent met the aforementioned HIS criteria (A-E) to be classified as a migraineur. The questionnaire was translated into Arabic and the questions were rephrased in everyday language if whenever needed. The IHS criteria subclassify people experiencing non-migraine headaches into tension-type and cluster headaches; however, this differentiation was not needed in our survey and would substantially add meaningless effort and time. Therefore, people 
Table 2. Prevalence of Migraine in Different Study Groups Sorted by Gender

\begin{tabular}{llllll}
\hline \multirow{2}{*}{ Diagnosis } & \multicolumn{3}{c}{ Female } & & \multicolumn{2}{c}{ Male } \\
\cline { 2 - 3 } \cline { 5 - 6 } & $\mathbf{N}$ & Prevalence (\%) & & N & Prevalence (\%) \\
\hline Migraineurs with aura & 26 & 16.3 & 13 & 6.7 \\
Migraineurs without aura & 10 & 6.3 & 1 & 0.5 \\
Headachers & 103 & 64.4 & 13.1 & 125 & 64.4 \\
Non-headachers & 21 & 100.0 & 55 & 28.4 \\
Total & 160 & & & 194 & 100.0 \\
\hline
\end{tabular}

reporting non-migraine headaches were classified as headaches. The third class of respondents were those who reported no headache in the past year (non-headaches).

\section{Data collection}

Surveys were conducted by local teams at each hospital. The survey packet contained a cover letter and a questionnaire with Arabic and English versions. The survey has been handed to each patient to read and decide whether to participate or not.

\section{Statistical analysis}

All collected data were transferred into a spreadsheet for further analysis. Standard descriptive measures were calculated for each question/item individually. For data analysis, SPSS 24 for Windows (SSPS Inc., Chicago, IL, USA) were used. Prevalence is expressed as a proportion and 95\% confidence intervals (CIs) were calculated for all patients with excluding those with any associated diseases from the analysis.
Migraineur status was modeled as a binary dependent variable (yes/no) using multiple logistic regression to examine associations with covariates of age, gender, habitat, marital status, employment status, educational level and smoking tobacco.

\section{Results}

Three hundred and fifty-four individuals were recruited to this study. Females constituted $54.3 \%$, and males constituted $45.7 \%$ of the recruited sample. Among the 354 studied individuals, the gender specific prevalence of headache was almost equal (64.4\%) among genders. However, aura occurred in $16.3 \%$ of females and only in $6.7 \%$ of males. The gender specific distribution of headache is depicted in Table 1, Table 2 , and Figure 1.

\section{Demographic variables among genders}

Table 3 shows the various demographic variables which were

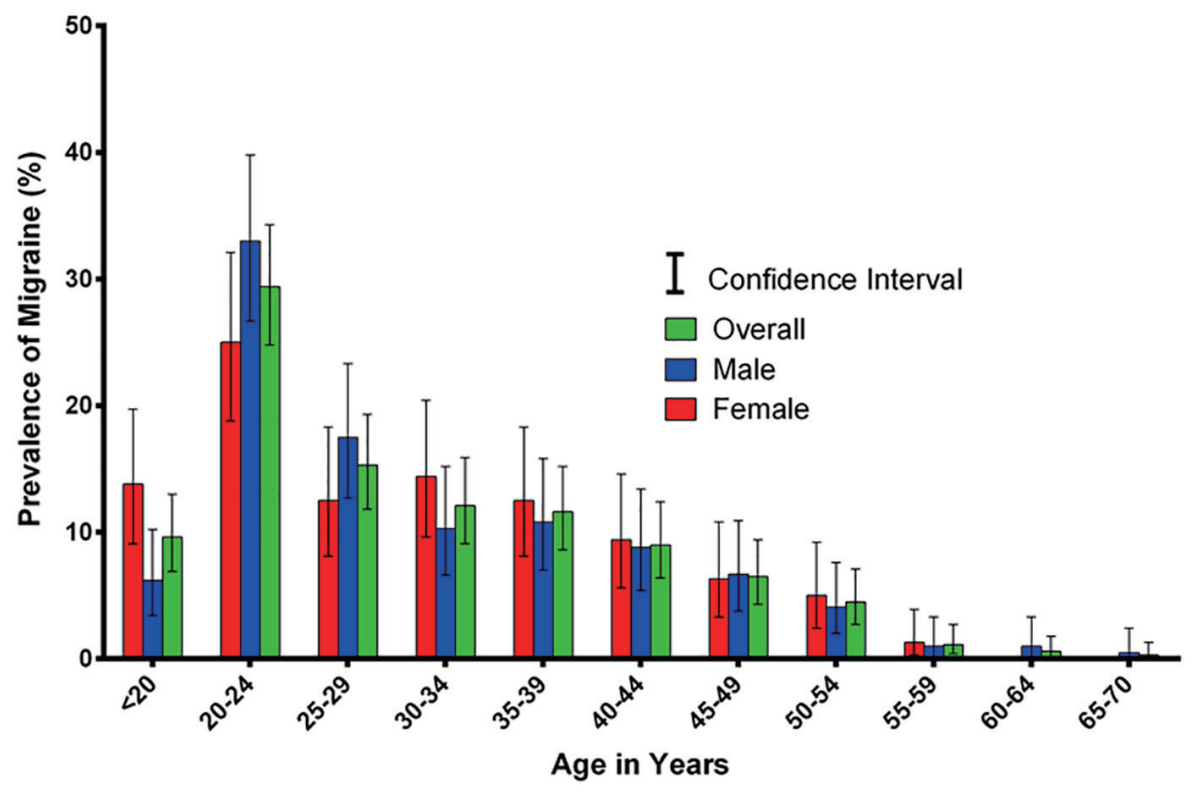

Figure 1. Sex-specific prevalence of migraine with associated $\mathrm{Cl}$ sorted by age. 
Table 3. Different Demographic Characteristics Among Study Participants

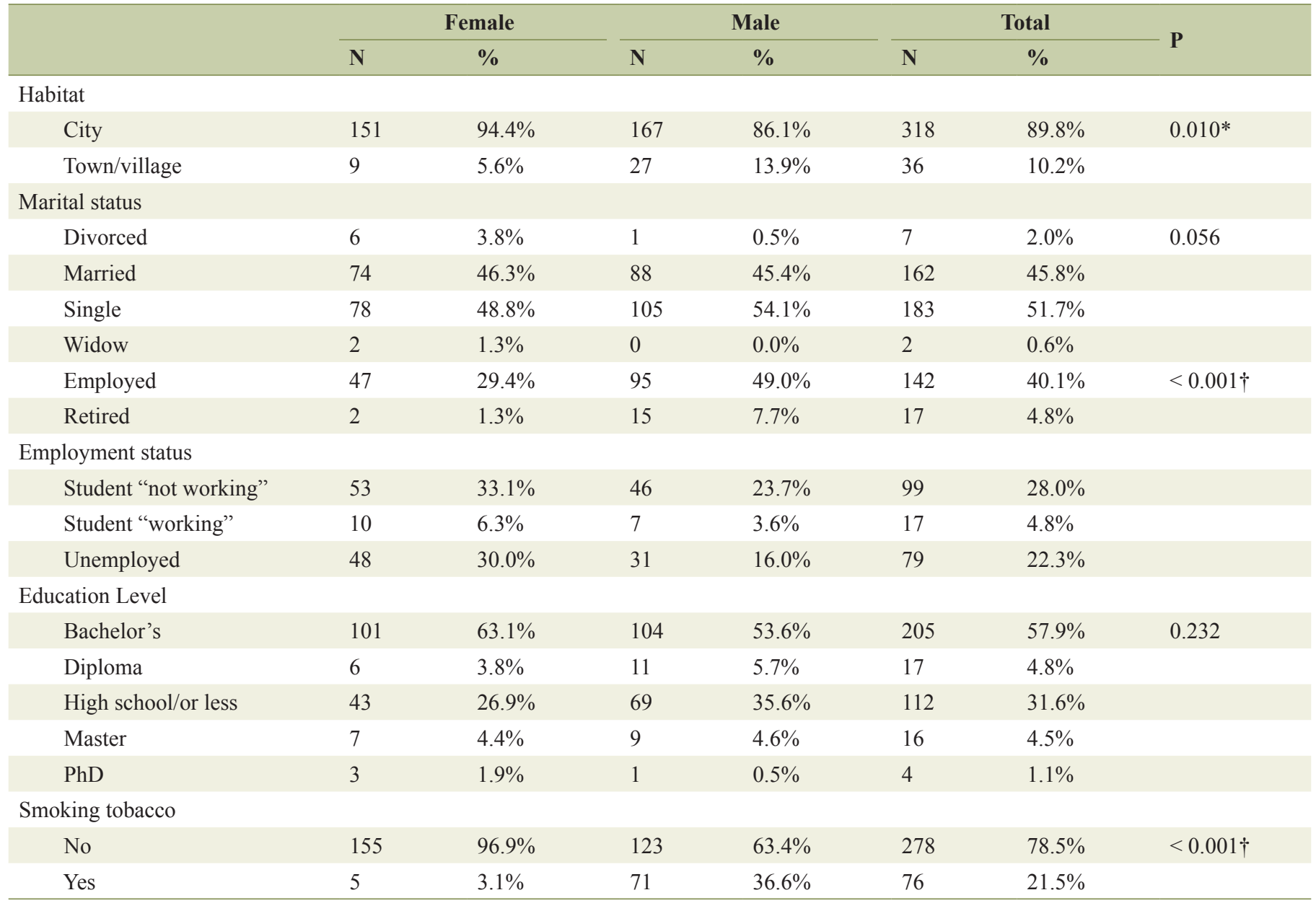

${ }^{*}$ The Chi-square statistic is significant $(P<0.05)$. †The Chi-square statistic is significant $(P<0.001) . N$ : number of observations.

studied; some of these showed statistically significant differences. The vast majority of studied individuals were city rather than country-side inhabitants, with figures of $89.8 \%$ and $10.2 \%$, respectively. Females living in cities $(94.4 \%)$ were significantly higher than males $(89.8 \%)(\mathrm{P}=0.010)$. No statistically significant difference was encountered as regards either the marital states or the educational level among studied subjects. Forty percent of participants were unemployed and $28 \%$ were non-working students. Among females, 29.4\% were unemployed and $30 \%$ were non-working students, whereas $16 \%$ and $23.7 \%$ of men were unemployed and non-working students, respectively $(\mathrm{P}<0.001)$. More than three-fourths $(78.5 \%)$ of participants were non-smokers, with smokers constituting $36.1 \%$ of males and $3.1 \%$ of females (P $<0.001)$.

\section{Migraine characteristics, medications usage, and response}

Migraine headache was experienced by $78.5 \%$ of studied subjects. Table 4 shows the characteristics of migraine headache, medications used, and outcome of treatment among males and females. Migraine headache was significantly more prevalent among females $(86.9 \%)$ than males $(71.6 \%)(\mathrm{P}=0.002)$. More than half of the recruited individuals reported that they had more than five previous attacks of headache, with values of $62.6 \%$ and $55.8 \%$ among females and males, respectively $(\mathrm{P}$ $<0.001)$. As regards the duration of headache, males seemed to have shorter headaches than females. Fifty-eight percent of males reported that their headache lasted less than $4 \mathrm{~h}$, whereas only $34.5 \%$ had such short headaches. Status migrainosus (headache lasting more than $72 \mathrm{~h}$ ) was more encountered among females with a figure of 5.6\% compared to only $1.4 \%$ among males. However, the differences were not statistically significant. The vast majority of the studied individuals reported pain scores ranging from 4 to 6 . Among females, $37.4 \%$, $32.4 \%$, and $15.8 \%$ reported pain scores of $4-6,6-9$, and $>$ 9 , respectively, whereas $54.7 \%, 22.3 \%$, and $5 \%$ of males reported scores of $4-6,6-9$, and $>9$, respectively $(P=0.002)$. The type of headache was also statistically different among the studied groups. Throbbing headache was reported by $49.6 \%$ and $56.8 \%$ of females and males, respectively. Next came the pressure headache with values of $46.6 \%$ and $42.4 \%$ among females and males, respectively $(\mathrm{P}=0.001)$. 
Table 4. Characteristics Migraine and Associated Diagnostics/Medications Among Study Participants

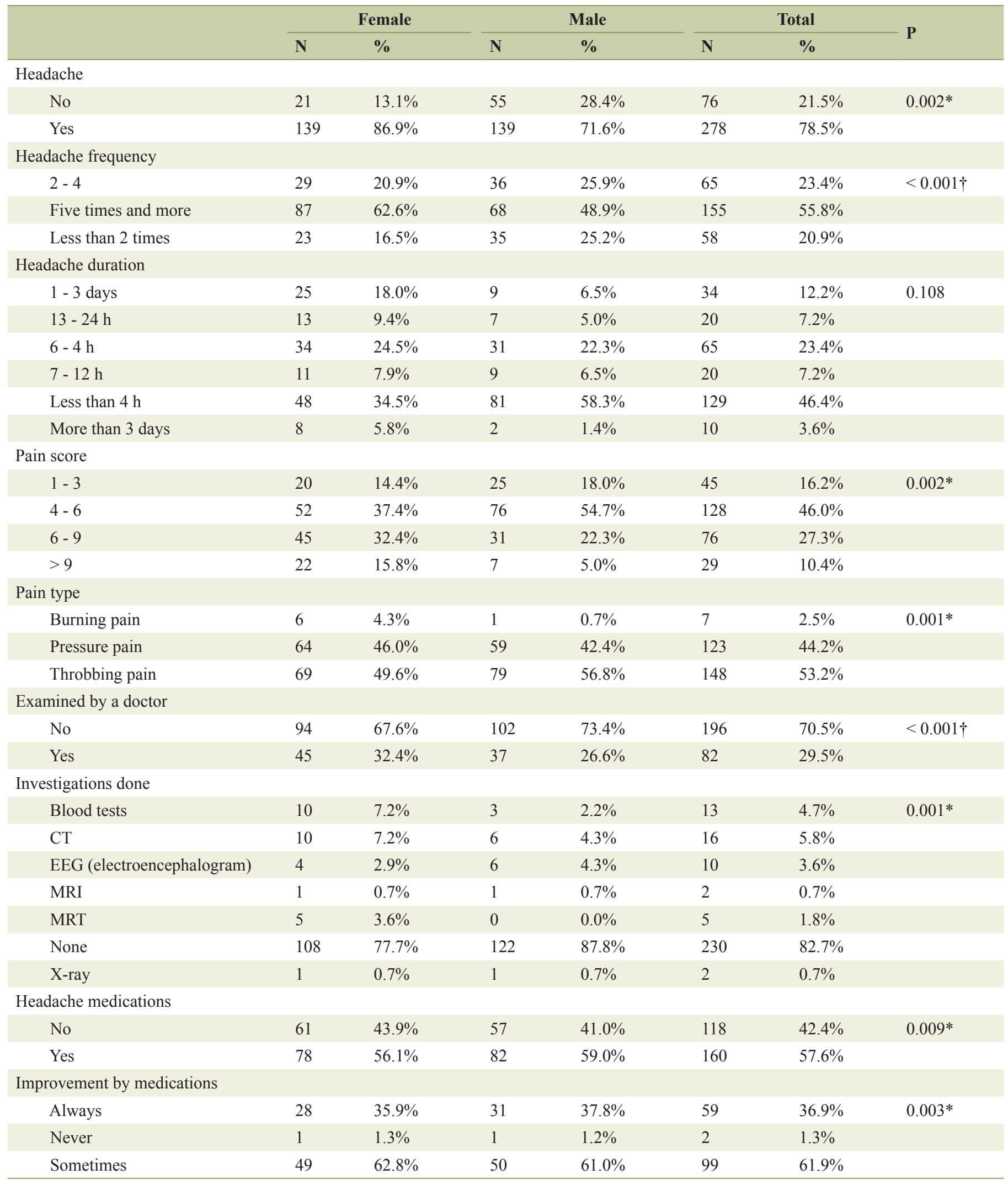

The Chi-square statistic is significant $(P<0.05)$. $†$ The Chi-square statistic is significant $(P<0.001)$. N: number of observations. 
Table 5. History and Nature of the Attacks Among Study Participants

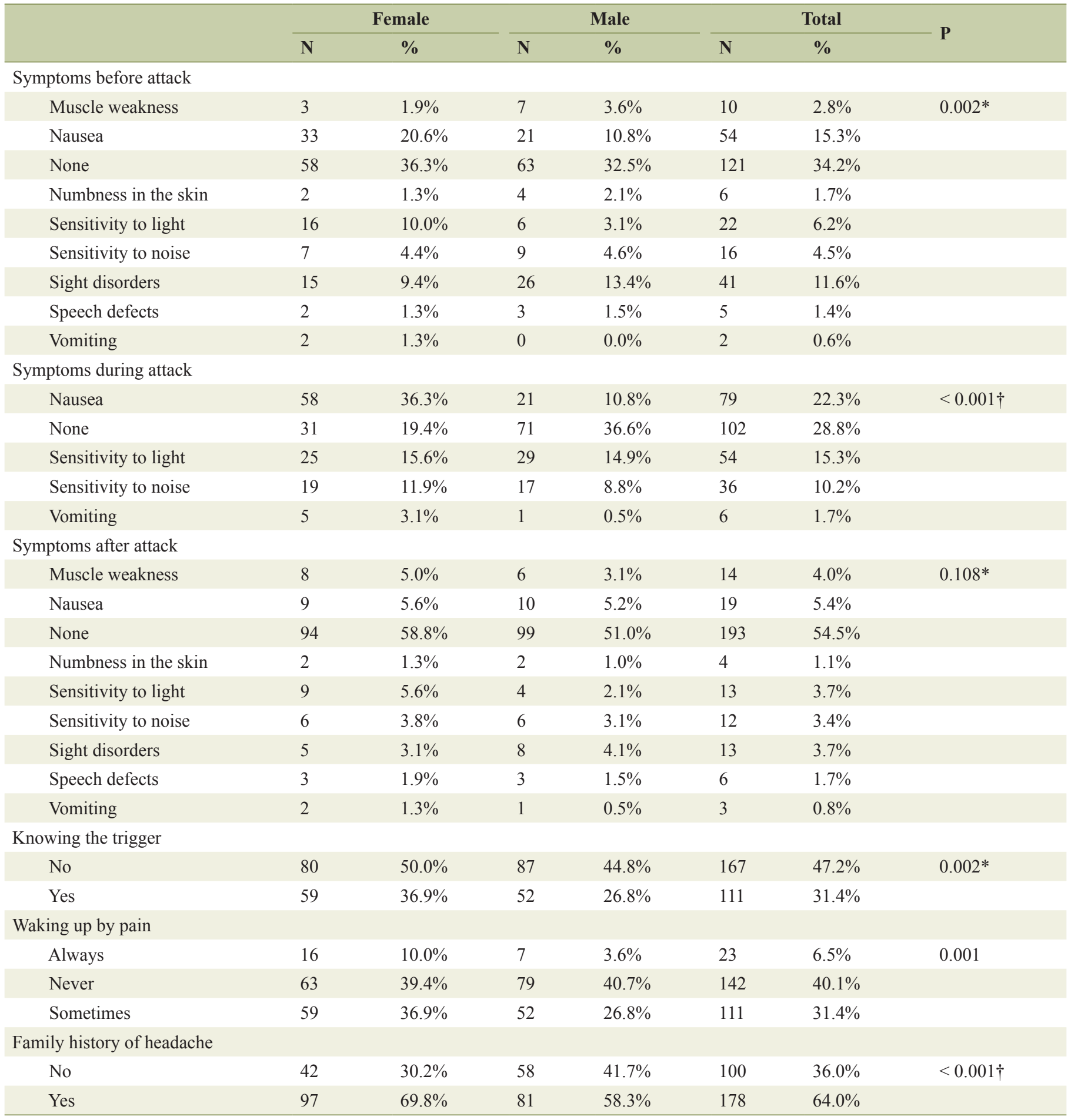

${ }^{*}$ The Chi-square statistic is significant $(P<0.05)$. $†$ The Chi-square statistic is significant $(P<0.001) . N$ : number of observations.

As regards medical advice seeking, less than one-third of the recruited subjects had seen doctors. Only $32.4 \%$ of females and $26.6 \%$ of males reported that they were seen by a doctor. Most of them did not have any investigation for their headache, with values of $77.7 \%$ of females and $87.8 \%$ of males. CT brain seemed to be the most common investigation done among $7 \%$ of females and $4.3 \%$ of males, followed by blood tests and EEG $(\mathrm{P}=0.001)$. Males received medications more than females; results of analysis showed that $56.1 \%$ of females and $59 \%$ of males received medications for their headache. Among 
Table 6. Logistic Regression Between Different Co-Variables and Migraine Prevalence

\begin{tabular}{lllll}
\hline Predictors & B & SE & Adjusted OR (95\% CI) $\dagger$ & P \\
\hline Age & -0.03 & 0.02 & $0.97(0.93-1.02)$ & 0.219 \\
Gender & -1.28 & 0.42 & $0.28(0.12-0.63)$ & $0.002 *$ \\
Habitat & -0.77 & 0.77 & $0.46(0.1-2.11)$ & 0.320 \\
Marital status & -0.46 & 0.37 & $0.63(0.3-1.3)$ & 0.211 \\
Employment status & -0.22 & 0.12 & $0.8(0.64-1.01)$ & 0.060 \\
Education level & 0.06 & 0.15 & $1.06(0.79-1.42)$ & 0.694 \\
Smoking tobacco & 0.01 & 0.51 & $1.01(0.37-2.76)$ & 0.978 \\
\hline
\end{tabular}

B: logistic regression coefficient; SE: standard error of logistic regression coefficient; OR: odds ratio; Cl: confidence interval; P: probability value. *Statistically significant $(<0.05)$. †Adjusted relative odds are odds ratio for migraineur status relative to the reference group (=1.0) for each covariate, after adjusting for all covariates by logistic regression.

males, $37.8 \%$ reported that the always improved, whereas only $35.9 \%$ of females reported so $(\mathrm{P}=0.003)$.

\section{Nature of migraine attacks}

Table 5 demonstrates the nature and characteristics of migraine attacks. The vast majority of patients reported no associated symptoms before, during, or after migraine attacks. However, many others reported nausea, vomiting, weakness, sensitivity to light, noise, sight disturbance, or speech problems. Nausea was found to be the most prominent manifestation experienced by patients before, during, and after the migraine attack. Before attack, $20.6 \%$ of females and $10.8 \%$ of males reported nauseating sensation. Nausea was also reported by $36.3 \%$ of females and $10.2 \%$ of males during the attack, and $5.6 \%$ of males and $5.2 \%$ of males after the attack. Sensitivity to light was as common as nausea after migraine attacks, which was reported by $5.6 \%$ and $2.1 \%$ of females and males, respectively. Vomiting, on the other hand, was the least symptom experienced before, during, or after the attack of migraine. It was reported by $1.3 \%$ of females before the attack, $3.1 \%$ of females and $1 \%$ of males during the attack, and $1.3 \%$ of females and $0.5 \%$ of males after the attack, respectively. Less than onethird of participants $(31.4 \%)$ reported that they could identify the migraine triggers; males could identify triggers more than females $(\mathrm{P}=0.0027)$. Forty-one percent of men reported that their headache had never awakened them from sleep, and $39.4 \%$ of females also reported so. Family history of migraine was reported among $69.8 \%$ of participating females and $58.3 \%$ of participating males.

\section{Correlation between demographic variables and migraine}

Logistic regression was performed to explore the correlation between different co-variables and migraine prevalence. Age, gender, habitat, marital status, employment status, educational level, and smoking were all studied. Gender was found to be the only demographic variable that was significantly correlated with migraine prevalence $(\mathrm{P}=0.002)$. The odds ratio was 0.28 indicating that males were only 0.28 times riskier of devel- oping migraine than females. Logistic regression of different variables is demonstrated in Table 6.

\section{Discussion}

Migraine headache is a common neurological disorder affecting Saudi population. The prevalence of headache in Taif city was found to be higher than prevalence reported in different studies. For instance, headache was reported in 53.2\% of individuals studied in Brazil in 2005 [7], 33.8\% in Nairobi [8], and $27.9 \%$ in Kuwait [9]. Females seemed to be significantly more affected than males $(\mathrm{P}=0.002)$, with an odds ratio of 0.28 for the gender difference. This finding is consistent with previous literature studies that stated that migraine headache was more prevalent among females $[7,10,11]$. High prevalence of migraine among females can be attributed not only to hormonal changes, but also to central cortical excitability $[12,13]$. Migraine headache was also found to be more prevalent among city habitants than countryside habitants. The stressful life in the city, and the lack of meditation and relaxing country nature can be the reasons behind the high prevalence of migraine among city inhabitants.

Migraine was significantly associated with high rates of unemployment $(\mathrm{P}<0.001)$. Up to one half of males and twothirds of females were either unemployed or non-working students. In agreement with our results, Stewart et al [14] reported in their study that migraine, especially chronic migraine, had a negative impact on employment, and that $19 \%$ of chronic migraineurs were less likely to be working for pay compared with episodic migraineurs. The severity of migraine attack can explain such negative impact on occupational aspects. Marital status, educational level, and tobacco smoking were not significantly correlated with migraine prevalence when logistic regression was performed.

Females and males reported their pain intensity differently. Although the vast majority of both genders reported that their pain score ranged from 4 to 6 , more females had scores from 6 to 9 , and scores more than $9(\mathrm{P}=0.002)$. Furthermore, less females reported that they improved after taking medications $(\mathrm{P}=0.003)$. This can be explained by the sex difference proved previously by Fillingim et al [15], in their review article, when 
they stated that males and females have different thresholds for pain sense and different response to analgesic medications. On the contrary, males seemed to be keener on seeking medical advice $(\mathrm{P}<0.001)$ and receiving medications $(\mathrm{P}=0.009)$. Females less interest in medications may be due to their lower response, their higher pain tolerance, socioeconomic issues, or other causes. More than three-fourths of the recruited sample did not undergo any investigation for their headache.

As regards the clinical presentation of headache, throbbing nature was experienced by around half of female and male participants, which is consistent with the nature of the classical migraine headache [3]. It never awake up to $40 \%$ of participants from sleep. More than one-third of recruited subjects reported no associated symptoms with migraine. Nausea, weakness, numbness, visual, and speech symptoms were reported and showed a significant gender difference. This goes in line with Buse et al [16] who observed significant gender differences in migraine associated symptomology, aura, and headache-related disability. Nausea was the most common experienced symptoms before, during, or after the headache in our study. In disagreement with us, Menon et al [17] reported that phonophobia and photophobia were more common than nausea among both males and females.

Only one-third of our studied participant had previous knowledge about migraine triggers, and females seemed to be more ignorant about them than males $(\mathrm{P}=0.002)$. This figure is close to the results reported by Wober et al [18] which indicated that migraineurs could identify less than half of the migraine triggers.

Family history of migraine was high among the studied population encountered in more than two-thirds of subjects. Females showed more positive family history of migraines than men. This is in agreement with previous literature studies that indicated high prevalence of positive family history among migraine patients $[11,19]$.

\section{Conclusions}

Migraine is prevalent in Taif city, Saudi Arabia. It is more prevalent among females, and among urban areas. Migraine causes negative consequences and is associated with high rates of unemployment. Most Saudi migraineurs encounter throbbing, moderately severe headache, that does not awake them from sleep. Females report more severe headaches, whereas men seek medical advice more and take medications. Nausea is the most common associated symptom with migraine. Only one-third of Saudi migraineurs possess a background knowledge about migraine triggers. Family history of migraine is very common among Saudi migraineurs.

\section{Acknowledgments}

The authors would like to thank Taif University ethical board for their cooperation and guidance through the whole process, and we like to thank all the patients that were part of this study, for taking their time to complete the survey.

\section{Competing Interests}

The authors declare that they have no competing interests.

\section{Grant Support}

None.

\section{References}

1. Steiner TJ, Stovner LJ, Vos T. GBD 2015: migraine is the third cause of disability in under 50s. J Headache Pain. 2016;17(1):104.

2. Noseda R, Burstein R. Migraine pathophysiology: anatomy of the trigeminovascular pathway and associated neurological symptoms, cortical spreading depression, sensitization, and modulation of pain. Pain. 2013;154(Suppl 1):S44-53.

3. Loder E, Weizenbaum E, Frishberg B, Silberstein S, American Headache Society Choosing Wisely Task F. Choosing wisely in headache medicine: the American Headache Society's list of five things physicians and patients should question. Headache. 2013;53(10):1651-1659.

4. H.L.L. Rima M Dafer. Migraine variants: overview, pathophysiology, epidemiology. 2017. [Online]. Available: https://emedicine.medscape.com/article/1142731-overview. [Accessed: 12-Nov-2017].

5. S. D. S. Shuhan Zhu. Status migrainosus. 2017. [Online]. Available: http://www.medlink.com/article/status_migrainosus. [Accessed: 12-Nov-2017].

6. Migraine with aura - ICHD-3 Beta the international classification of headache disorders 3rd edition (Beta version). [Online]. Available: https://www.ichd-3.org/1migraine/1-2-migraine-with-aura/. [Accessed: 12-Nov2017].

7. Domingues RB, Aquino CC, Santos JG, da Silva AL, Kuster GW. Prevalence and impact of headache and migraine among Pomeranians in Espirito Santo, Brazil. Arq Neuropsiquiatr. 2006;64(4):954-957.

8. Amayo EO, Jowi JO, Njeru EK. Headache associated disability in medical students at the Kenyatta National Hospital, Nairobi. East Afr Med J. 2002;79(10):519-523.

9. Al-Hashel JY, Ahmed SF, Alroughani R, Goadsby PJ. Migraine among medical students in Kuwait University. J Headache Pain. 2014;15:26.

10. Mengistu G, Alemayehu S. Prevalence and burden of primary headache disorders among a local community in Addis Ababa, Ethiopia. J Headache Pain. 2013;14:30.

11. Ofovwe GE, Ofili AN. Prevalence and impact of headache and migraine among secondary school students in Nigeria. Headache. 2010;50(10):1570-1575.

12. Sacco S, Ricci S, Degan D, Carolei A. Migraine in women: the role of hormones and their impact on vascular diseases. J Headache Pain. 2012;13(3):177-189.

13. Goadsby PJ. Pathophysiology of migraine. Ann Indian Acad Neurol. 2012;15(Suppl 1):S15-22. 
14. Stewart WF, Wood GC, Manack A, Varon SF, Buse DC, Lipton RB. Employment and work impact of chronic migraine and episodic migraine. J Occup Environ Med. 2010;52(1):8-14.

15. Fillingim RB, King CD, Ribeiro-Dasilva MC, RahimWilliams B, Riley JL, 3rd. Sex, gender, and pain: a review of recent clinical and experimental findings. J Pain. 2009;10(5):447-485.

16. Buse DC, Loder EW, Gorman JA, Stewart WF, Reed ML, Fanning KM, Serrano D, et al. Sex differences in the prevalence, symptoms, and associated features of migraine, probable migraine and other severe headache: results of the American Migraine Prevalence and Preven- tion (AMPP) Study. Headache. 2013;53(8):1278-1299.

17. Menon B, Kinnera N. Prevalence and characteristics of migraine in medical students and its impact on their daily activities. Ann Indian Acad Neurol. 2013;16(2):221-225.

18. Wober C, Holzhammer J, Zeitlhofer J, Wessely P, WoberBingol C. Trigger factors of migraine and tension-type headache: experience and knowledge of the patients. J Headache Pain. 2006;7(4):188-195.

19. Garah M, Neyaz H, Shaqrun F, Alhussaini K, Hafiz B, Alrehaili M, Zahid B, Alraddadi W, Aboauf H, Albouq N, Alhejaili M. Prevalence of migraine among female students at Taibah University, Kingdom of Saudi Arabia. Int J Adv Res. 2016;4(7):1526-1534. 